\title{
Intraoperative Injection of Technetium-99m Sulfur Colloid for Sentinel Lymph Node Biopsy in Breast Cancer Patients: A Single Institution Experience
}

\author{
Julian Berrocal, ${ }^{1}$ Lawrence Saperstein, ${ }^{2}$ Baiba Grube, ${ }^{1}$ Nina R. Horowitz, ${ }^{1}$ \\ Anees B. Chagpar, ${ }^{1}$ Brigid K. Killelea, ${ }^{1}$ and Donald R. Lannin ${ }^{1}$ \\ ${ }^{1}$ Department of Surgery, Yale University School of Medicine and Yale Comprehensive Cancer Center, \\ New Haven, CT 06520, USA \\ ${ }^{2}$ Department of Radiology and Biomedical Imaging, Yale University School of Medicine and Yale Comprehensive Cancer Center, \\ New Haven, CT 06520, USA
}

Correspondence should be addressed to Donald R. Lannin; donald.lannin@yale.edu

Received 18 February 2017; Accepted 2 April 2017; Published 13 April 2017

Academic Editor: William Dooley

Copyright (C) 2017 Julian Berrocal et al. This is an open access article distributed under the Creative Commons Attribution License, which permits unrestricted use, distribution, and reproduction in any medium, provided the original work is properly cited.

\begin{abstract}
Background. Most institutions require a patient undergoing sentinel lymph node biopsy to go through nuclear medicine prior to surgery to be injected with radioisotope. This study describes the long-term results using intraoperative injection of radioisotope. Methods. Since late 2002, all patients undergoing a sentinel lymph node biopsy at the Yale-New Haven Breast Center underwent intraoperative injection of technetium-99m sulfur colloid. Endpoints included number of sentinel and nonsentinel lymph nodes obtained and number of positive sentinel and nonsentinel lymph nodes. Results. At least one sentinel lymph node was obtained in 2,333 out of 2,338 cases of sentinel node biopsy for an identification rate of $99.8 \%$. The median number of sentinel nodes found was 2 and the mean was 2.33 (range: 1-15). There were 512 cases (21.9\%) in which a sentinel node was positive for metastatic carcinoma. Of the patients with a positive sentinel lymph node who underwent axillary dissection, there were 242 cases (54.2\%) with no additional positive nonsentinel lymph nodes. Advantages of intraoperative injection included increased comfort for the patient and simplification of scheduling. There were no radiation related complications. Conclusion. Intraoperative injection of technetium-99m sulfur colloid is convenient, effective, safe, and comfortable for the patient.
\end{abstract}

\section{Introduction}

Sentinel lymph node biopsy has replaced axillary dissection for axillary staging in clinically node negative breast cancer patients [1]. Although this is considered to be standard of care and there are guidelines and recommendations on how to perform a sentinel lymph node biopsy, there is no standardized protocol for the procedure. There is variability as to which dyes are used, the amount of dye injected, and the timing of injection. When the procedure was first developed in the 1990s, radioisotope was injected into the peritumoral area of the breast and lymphoscintigraphy was performed to evaluate lymphatic drainage into the sentinel nodes over the next hour or two. However, subsequent studies showed that intradermal and periareolar injection of the isotope resulted in faster uptake into the nodes and yielded a higher identification rate [2-6]. Furthermore, it was found that routine lymphoscintigraphy was not necessary as accurate identification of sentinel nodes was possible without the preoperative images [7]. This made intraoperative injection a particularly feasible approach and several small series reported excellent results [8-10]. These series showed clearly that intraoperative injection resulted in decreased patient discomfort and markedly improved efficiency of scheduling for both the patient and surgeon. Despite clear advantages to intraoperative injection, many institutions still require patients to go through nuclear medicine for injection prior to surgery. At Yale, we have used intraoperative injection of 


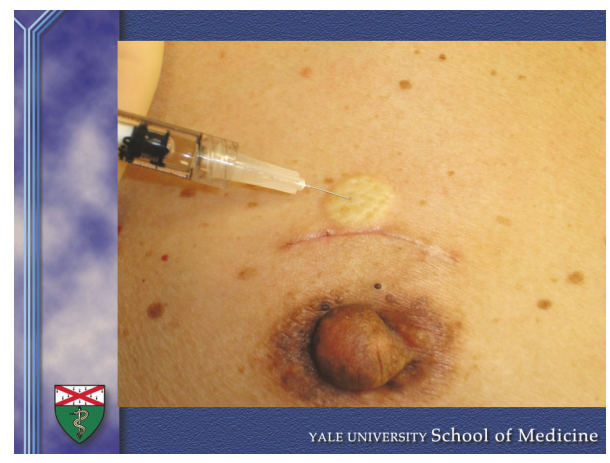

FIGURE 1: Intradermal injection of Tc-99m above excisional biopsy scar.

technetium-99m sulfur colloid for all sentinel node biopsies since late 2002. The aim of this study is to describe our longterm experience with the technique in more than 2,300 breast cancer patients.

\section{Methods}

2.1. Sentinel Lymph Node Procedure. On the day of surgery, a nuclear medicine technologist delivers a lead case to the operating room containing $0.25-0.5 \mathrm{mCi}$ of technetium- $99 \mathrm{~m}$ sulfur colloid in a $0.4 \mathrm{~mL}$ volume. After induction of general anesthesia, the radioactive tracer is infiltrated intradermally as a skin wheal directly over the breast cancer site or injected into the subdermal plexus of the areola. In rare circumstances in which there is a scar between the areola and the axilla, the radioisotope is injected lateral to the scar so as to avoid interference from scar tissue (see Figure 1). The surgeon then preps and drapes the patient in the usual manner. If dual tracer is desired by the surgeon, isosulfan blue or diluted methylene blue dye is also injected either peritumorally or into the subareolar plexus. About 10 to 15 minutes after injection of isotope, a hot spot can be identified in the axilla with a gamma probe and a small incision made over it. After entering the clavipectoral fascia, the gamma probe is used to identify the hottest node and any other hot nodes demonstrating greater than $10 \%$ of counts of the hottest node or any node that is blue or found to feel suspicious. The sentinel lymph nodes are removed and sent to pathology for frozen section or for permanent section based on surgeon preference.

\subsection{Nuclear Medicine Protocol for Intraoperative Technetium} Injection. The licensed nuclear radiology attending physician authorizes release of technetium for intraoperative injection. The surgeons have received instruction on how to properly handle the isotope. For each case where SLNB is scheduled, nuclear medicine staff delivers Tc-99m sulfur colloid in a lead box from the department of nuclear medicine to the appropriate operating room. The surgeon then carefully injects the Tc-99m sulfur colloid while wearing gloves and eye protection. The empty syringe, needle, and any gauze or alcohol wipes that come into contact with the isotope are immediately placed back into the lead box, which is then taken back to the nuclear medicine department for proper disposal. A radiation safety officer is available at all times for questions or to respond to spills. To date, there have been no radioactive spills in the operating room or any inappropriate disposal of radioactive waste. Surgeons and OR staff are not required to wear radioactive monitoring badges given the limited exposure.

2.3. Data Collection and Statistics. The Breast Center maintains a prospectively collected database which contains data on all patients undergoing oncologic breast surgery. After approval by the Yale University Human Investigations Committee (HIC), patient deidentified data was abstracted from this database on all patients undergoing a sentinel lymph node biopsy for breast cancer between 2003 and 2014. Demographic data collected included patient age, ethnicity, cancer histology, molecular subtype, TNM stage, and breast surgical procedure, and endpoints collected included the number of sentinel lymph nodes obtained, number of positive sentinel lymph nodes, number of nonsentinel lymph nodes obtained, and number of positive nonsentinel lymph nodes. Because the data was merely descriptive, no statistical comparisons were needed.

\section{Results}

There were 2,333 patients in the database who underwent a sentinel lymph node biopsy. All patients had radioisotope injection of technetium-99m sulfur colloid and approximately $56 \%$ of cases had dual tracer with both the radioisotope and blue dye. During the early part of the time period, there were 5 additional cases where a sentinel node biopsy was attempted but no node was found, for an identification rate of $99.8 \%$. All of the five cases where a node was not found had an extensive scar between the injection site and the axilla. This leads to our current practice of injecting the isotope in a location to avoid interference with a surgical scar. We also had a number of cases where a second sentinel node biopsy was performed after a previous sentinel node biopsy, but these cases were excluded from this analysis.

3.1. Patient Demographics. The patient demographics for all 2,333 patients are shown in Table 1 . The majority of patients were Caucasian (80.3\%), with Blacks representing $9.5 \%$ of the study population. The average patient age was 56.9 years, with $77.5 \%$ of the patient population in the age range of $41-70$ years. Most cancers were infiltrating ductal carcinoma (67.4\%), with ductal carcinoma in situ (DCIS) and infiltrating lobular carcinoma representing $10.8 \%$ and $10.4 \%$, respectively. The molecular subtype of tumors most represented in the study population was ER/PR positive and Her-2 negative (64\%). Over $94 \%$ of the cancers in this study had an early $\mathrm{T}$ stage (DCIS or stage I/II) and the majority (47.6\%) were moderately differentiated. Partial mastectomy was more commonly performed than total mastectomy $(54.7 \%$ versus $45.3 \%)$. This rate of mastectomy is higher than our overall 
TABLE 1: Demographic data.

\begin{tabular}{|c|c|c|}
\hline & Frequency & Percent \\
\hline \multicolumn{3}{|l|}{ Ethnicity } \\
\hline Caucasian & 1,874 & 80.3 \\
\hline Black & 222 & 9.5 \\
\hline Hispanic & 113 & 4.8 \\
\hline Asian & 66 & 2.8 \\
\hline Other & 58 & 2.5 \\
\hline \multicolumn{3}{|l|}{ Age (years) } \\
\hline$<30$ & 22 & 0.9 \\
\hline $31-40$ & 164 & 7 \\
\hline $41-50$ & 608 & 26.1 \\
\hline $51-60$ & 649 & 27.8 \\
\hline $61-70$ & 553 & 23.7 \\
\hline$>71$ & 337 & 14.4 \\
\hline \multicolumn{3}{|l|}{ Tumor Histology } \\
\hline Infiltrating ductal & 1,573 & 67.4 \\
\hline Infiltrating lobular & 242 & 10.4 \\
\hline Mixed ductal and lobular & 137 & 5.9 \\
\hline Ductal carcinoma in situ & 253 & 10.8 \\
\hline Other & 128 & 5.5 \\
\hline \multicolumn{3}{|l|}{ Molecular subtype } \\
\hline ER/PR positive, Her-2 negative & 1,494 & 64 \\
\hline ER/PR positive, Her-2 positive & 160 & 6.9 \\
\hline ER/PR negative, Her-2 positive & 102 & 4.4 \\
\hline ER/PR negative, Her-2 negative & 243 & 10.4 \\
\hline \multicolumn{3}{|l|}{ T stage } \\
\hline 0 & 253 & 10.8 \\
\hline 1 & 1,366 & 58.6 \\
\hline 2 & 592 & 25.4 \\
\hline 3 & 111 & 4.8 \\
\hline 4 & 11 & 0.5 \\
\hline \multicolumn{3}{|l|}{ Tumor grade } \\
\hline Well differentiated & 499 & 21.4 \\
\hline Moderately differentiated & 1,110 & 47.6 \\
\hline Poorly differentiated & 557 & 23.9 \\
\hline \multicolumn{3}{|l|}{ Surgery } \\
\hline Partial mastectomy & 1,277 & 54.7 \\
\hline $\begin{array}{l}\text { Mastectomy (including bilateral, } \\
\text { simple, and modified radical) }\end{array}$ & 1,056 & 45.3 \\
\hline
\end{tabular}

rate because our practice has been to perform sentinel node biopsy on patients with DCIS who undergo mastectomy but not on most DCIS patients undergoing lumpectomy. Thus, DCIS patients receiving lumpectomy would not be included in this analysis.

3.2. Sentinel Lymph Nodes. The median number of sentinel nodes obtained was 2 and the mean number was 2.33 with a range of 1-15 nodes. About a third of cases had only one
TABLE 2: Sentinel lymph nodes obtained.

\begin{tabular}{lcc}
\hline & Frequency & Percent \\
\hline 1 & 779 & 33.4 \\
2 & 754 & 32.3 \\
$\geq 3$ & 800 & 34.3 \\
Total & 2,333 & 100 \\
\hline
\end{tabular}

TABLE 3: Positive sentinel lymph nodes.

\begin{tabular}{lcc}
\hline & Frequency & Percent \\
\hline 0 & 1,821 & 78.1 \\
1 & 365 & 15.6 \\
2 & 101 & 4.3 \\
$\geq 3$ & 46 & 2 \\
Total & 2,333 & 100 \\
\hline
\end{tabular}

TABLE 4: Positive sentinel lymph nodes by histology.

\begin{tabular}{lcc}
\hline & Invasive carcinoma & Ductal carcinoma in situ \\
\hline 0 & $1,569(75.4 \%)$ & $252(99.6 \%)$ \\
1 & $365(17.5 \%)$ & $0(0 \%)$ \\
2 & $100(4.8 \%)$ & $1(0.4 \%)$ \\
$\geq 3$ & $46(2.2 \%)$ & $0(0 \%)$ \\
Total & 2,080 & 253 \\
\hline
\end{tabular}

sentinel lymph node removed, a third had 2, and a third had 3 or more (Table 2). Of all the sentinel lymph nodes obtained, only 512 cases (21.9\%) were positive for metastatic carcinoma as shown in Table 3. In the subset of patients with invasive carcinoma, $24.6 \%$ had at least one positive sentinel lymph node (Table 4). However, of the patients with in situ carcinoma, there was only one case which had a positive sentinel lymph node. There were other cases with a positive sentinel node where the preoperative diagnosis based on the needle biopsy was DCIS, but all of these cases except for one were upgraded to invasive breast cancer on the final pathology.

3.3. Nonsentinel Lymph Nodes. Table 5 shows the number of nonsentinel lymph nodes obtained following a positive sentinel lymph node biopsy. Until 2011, almost all of our patients who had a positive sentinel node underwent axillary dissection. However, following the publication of the ACOSOG Z11 trial [11], there were 87 cases $(16.3 \%$ of the entire group) in which no further lymph nodes were excised. Of the patients with a positive sentinel lymph node biopsy who underwent a completion axillary lymph node dissection, 242 cases $(54.2 \%)$ had no additional positive lymph nodes as shown in Table 6.

\section{Discussion}

Although historically an axillary lymph node dissection was performed to stage the axilla, compared to sentinel lymph node biopsy, there was increased morbidity including lymphedema, nerve disruption, chronic shoulder pain, weakness, 
TABLE 5: Nonsentinel lymph nodes obtained in sentinel lymph node positive patients.

\begin{tabular}{lcc}
\hline Nonsentinel lymph nodes & Frequency & Percent \\
\hline 0 & 87 & 16.3 \\
$1-10$ & 203 & 38 \\
$11-20$ & 199 & 37.4 \\
$21-30$ & 68 & 7.8 \\
$31-39$ & 4 & 0.8 \\
\hline
\end{tabular}

TABLE 6: Positive nonsentinel lymph nodes following positive sentinel lymph node biopsy.

\begin{tabular}{lcc}
\hline $\begin{array}{l}\text { Positive nonsentinel lymph } \\
\text { nodes }\end{array}$ & $\begin{array}{l}\text { Cases with a positive } \\
\text { sentinel lymph node }\end{array}$ & Percent \\
\hline 0 & 242 & 54.2 \\
1 & 65 & 14.5 \\
2 & 42 & 9.4 \\
3 & 22 & 4.9 \\
4 & 17 & 3.8 \\
$5-10$ & 38 & 8.4 \\
$11-20$ & 15 & 3.2 \\
$21-29$ & 6 & 1.2 \\
\hline
\end{tabular}

and joint dysfunction. As a result, sentinel lymph node biopsy has become standard of care to allow for proper axillary lymph node staging $[1,12]$. This is usually performed with radioactive Tc-99m labeled sulfur colloid [13], vital blue dye such as isosulfan blue or methylene blue [14], or the combination $[15,16]$. Studies performed during the learning curve for the procedure usually show a higher identification rate using both blue dye and radioisotope in combination $[16,17]$. However, there are occasional allergic reactions to the blue dyes, including anaphylactic reactions, urticaria, rash, blue hives, and pruritus associated with isosulfan blue dye and skin necrosis associated with nondiluted methylene blue. Many surgeons have found that, with extensive experience, the blue dye is not always necessary and can be used selectively in the rare cases where radioactivity is not detected in a node. The results of our current study show that it is possible to get essentially a $100 \%$ identification rate either with the combination of isotope and blue dye or with isotope alone.

With the speed of lymph node localization provided by intradermal and subareolar injection [2-6] and the realization that lymphoscintigraphy images are not needed [7], there is no reason not to inject the isotope in the operating room after induction of anesthesia. Several small series have shown that this provides excellent results. A study by Layeeque et al. [9] demonstrated successful use of intraoperative subareolar technetium-99m sulfur colloid and blue dye. Ninety-six sentinel lymph node biopsy procedures were performed in 88 breast cancer patients, and $97 \%$ percent of the cases demonstrated a "hot" sentinel lymph node. Similar to our experience, the three percent of patients that did not have successful localization had a history of prior surgery between the injection site and the axilla. Zogakis et al. [10] used intraoperative subareolar injection of Tc-99m labeled sulfur colloid along with blue dye in 122 patients and found a sentinel node in 99.2\%. Dauphine et al. [8] injected 100 consecutive patients intraoperatively with Tc-99m labeled sulfur colloid immediately after induction of anesthesia and compared this group to the previous 100 patients who had been injected preoperatively. The sentinel node identification rate was $100 \%$ in the intraoperative group compared to $96 \%$ in the preoperative group; there was no difference in the number of nodes found or the percent of nodes that were positive for metastases. The current report of 2,333 patients injected intraoperatively represents the largest series in the literature.

The benefits of intraoperative injection in our experience include both patient and surgeon related factors as outlined in Box 1. From a patient standpoint, pain at the time of radioisotope injection was one of the most common complaints with preoperative injection. Injected local anesthesia was not given as it could impact the uptake of the radioisotope within the lymphatics. From a logistical standpoint, patients do not have to travel to other departments on the day of surgery if an intraoperative injection of radioisotope is performed. From a surgical scheduling standpoint, intraoperative injection allows greater flexibility as a case can start early in the morning, there does not need to be coordinated scheduling between surgery and nuclear medicine, and there are never any surgical delays due to patients being held longer than expected in nuclear medicine.

It is important to point out that intraoperative injection requires collaboration between the surgeons and nuclear radiologists. Ultimately the nuclear radiologist bears the responsibility for the safe use of the isotopes. From a radiation safety standpoint, Miner et al. [18] demonstrated that the procedure is safe. A surgeon could safely perform 1,000 procedures per year without surpassing the OSHA defined safety limit. Stratmann et al. [19] conducted a study to investigate radiation exposure to the surgeon, scrub nurse, pathologist, and $\mathrm{OR}$ equipment to determine safety of sentinel lymph node biopsy with radioisotope. They concluded that a primary surgeon could perform 2,190 hours, a scrub nurse 33,333 hours, and a pathologist 14,705 hours of procedural work with radioisotope annually before surpassing radiation safety limits set forth by OSHA. Furthermore, operative instruments, pathology slides, and cryostat machines require no special handling following a sentinel lymph node biopsy with radioisotope. In the present study, all radioactive material was placed into a lead case during delivery to the operating suite and all material which contacted the radioactive isotope was returned to nuclear medicine within the lead case. No complications occurred as a result of the radioactive isotope.

We are not advocating that intraoperative injection should become a standard of care that is used everywhere. Perhaps at institutions with a large number of low volume breast surgeons, intraoperative injection may not be feasible. Each institution should decide what works best for its particular situation. However, at Yale, with a small number of high volume breast surgeons, intraoperative injection works very well. 
(1) Patient factors

(i) Decreased discomfort as patient is under anesthesia

(ii) Improved logistics as patient does not need to go to nuclear medicine

(2) Surgeon factors

(i) Can have a first case start time and no case delay due to nuclear medicine schedule

Box 1: Advantages of intraoperative radioisotope injection.

\section{Conclusion}

In our long-term, prospective experience, intraoperative injection of the radioisotope, technetium-99m sulfur colloid is convenient, effective, safe, and comfortable for the patient. The sentinel lymph node detection rate was essentially $100 \%$.

\section{Conflicts of Interest}

The authors declare that there are no conflicts of interest regarding the publication of this paper.

\section{References}

[1] E. A. Newman and L. A. Newman, "Lymphatic mapping techniques and sentinel lymph node biopsy in breast cancer," Surgical Clinics of North America, vol. 87, no. 2, pp. 353-364, 2007.

[2] T. W. Kersey, J. Van Eyk, D. R. Lannin, A. N. Chua, and L. Tafra, "Comparison of intradermal and subcutaneous injections in lymphatic mapping," Journal of Surgical Research, vol. 96, no. 2, pp. 255-259, 2001.

[3] K. M. Lin, T. H. Patel, A. Ray et al., "Intradermal radioisotope is superior to peritumoral blue dye or radioisotope in identifying breast cancer sentinel nodes," Journal of the American College of Surgeons, vol. 199, no. 4, pp. 561-566, 2004.

[4] F. J. Fleming, A. D. K. Hill, D. Kavanagh et al., "Intradermal radioisotope injection optimises sentinel lymph node identification in breast cancer," European Journal of Surgical Oncology, vol. 29, no. 10, pp. 835-838, 2003.

[5] D. C. Linehan, A. D. K. Hill, T. Akhurst et al., "Intradermal radiocolloid and intraparenchymal blue dye injection optimize sentinel node identification in breast cancer patients," Annals of Surgical Oncology, vol. 6, no. 5, pp. 450-454, 1999.

[6] S. P. Povoski, J. O. Olsen, D. C. Young et al., "Prospective randomized clinical trial comparing intradermal, intraparenchymal, and subareolar injection routes for sentinel lymph node mapping and biopsy in breast cancer," Annals of Surgical Oncology, vol. 13, no. 11, pp. 1412-1421, 2006.

[7] K. M. McMasters, S. L. Wong, T. M. Tuttle et al., "Preoperative lymphoscintigraphy for breast cancer does not improve the ability to identify axillary sentinel lymph nodes," Annals of Surgery, vol. 231, no. 5, pp. 724-731, 2000.

[8] C. E. Dauphine, I. Khalkhali, M. P. Vargas, N. M. Isaac, J. Haukoos, and H. I. Vargas, "Intraoperative injection of technetium-99m sulfur colloid is effective in the detection of sentinel lymph nodes in breast cancer," American Journal of Surgery, vol. 192, no. 4, pp. 423-426, 2006.

[9] R. Layeeque, J. Kepple, R. S. Henry-Tillman et al., "Intraoperative subareolar radioisotope injection for immediate sentinel lymph node biopsy," Annals of Surgery, vol. 239, no. 6, pp. 841$848,2004$.

[10] T. G. Zogakis, R. E. Wetherille, R. D. Christensen et al., "Intraoperative subareolar injection of $99 \mathrm{mTc}$-labeled sulfur colloid results in consistent sentinel lymph node identification," Annals of Surgical Oncology, vol. 12, no. 2, pp. 167-172, 2005.

[11] A. E. Giuliano, K. K. Hunt, K. V. Ballman et al., "Axillary dissection vs no axillary dissection in women with invasive breast cancer and sentinel node metastasis: a randomized clinical trial," JAMA, vol. 305, no. 6, pp. 569-575, 2011.

[12] D. N. Krag, S. J. Anderson, T. B. Julian et al., "Sentinellymph-node resection compared with conventional axillarylymph-node dissection in clinically node-negative patients with breast cancer: overall survival findings from the NSABP B-32 randomised phase 3 trial," The Lancet Oncology, vol. 11, no. 10, pp. 927-933, 2010.

[13] D. N. Krag, D. L. Weaver, J. C. Alex, and J. T. Fairbank, "Surgical resection and radiolocalization of the sentinel lymph node in breast cancer using a gamma probe," Surgical Oncology, vol. 2, no. 6, pp. 335-340, 1993.

[14] A. E. Giuliano, D. M. Kirgan, J. M. Guenther, and D. L. Morton, "Lymphatic mapping and sentinel lymphadenectomy for breast cancer," Annals of Surgery, vol. 220, no. 3, pp. 391-401, 1994.

[15] K. M. K. McMasters, T. M. Tuttle, D. J. Carlson et al., "Sentinel lymph node biopsy for breast cancer: a suitable alternative to routine axillary dissection in multi-institutional practice when optimal technique is used," Journal of Clinical Oncology, vol. 18, no. 13, pp. 2560-2566, 2000.

[16] L. Tafra, D. R. Lannin, M. S. Swanson et al., "Multicenter trial of sentinel node biopsy for breast cancer using both technetium sulfur colloid and isosulfan blue dye," Annals of Surgery, vol. 233, no. 1, pp. 51-59, 2001.

[17] P.-S. He, F. Li, G.-H. Li, C. Guo, and T.-J. Chen, "The combination of blue dye and radioisotope versus radioisotope alone during sentinel lymph node biopsy for breast cancer: a systematic review," BMC Cancer, vol. 16, no. 1, article 107, 2016.

[18] T. J. Miner, C. D. Shriver, P. R. Flicek et al., "Guidelines for the safe use of radioactive materials during localization and resection of the sentinel lymph node," Annals of Surgical Oncology, vol. 6, no. 1, pp. 75-82, 1999.

[19] S. L. Stratmann, T. M. McCarty, and J. A. Kuhn, "Radiation safety with breast sentinel node biopsy," American Journal of Surgery, vol. 178, no. 6, pp. 454-457, 1999. 


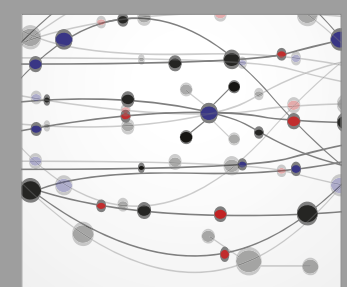

The Scientific World Journal
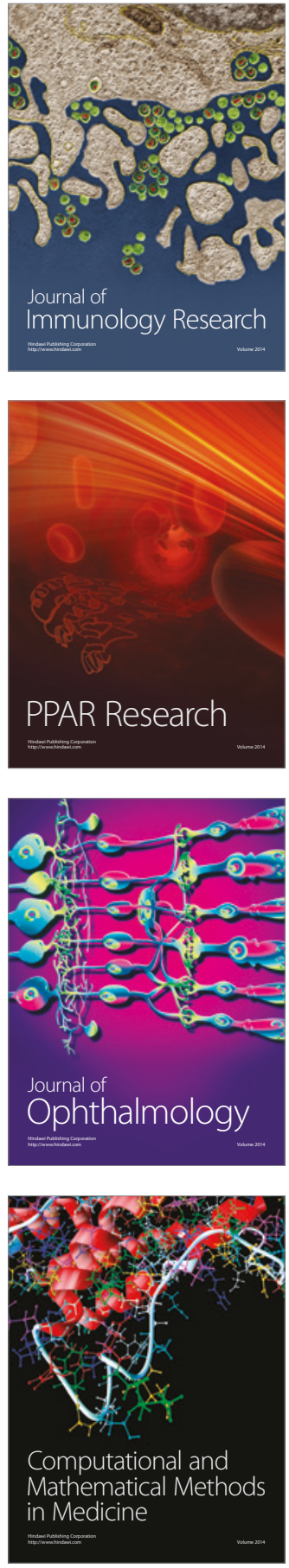

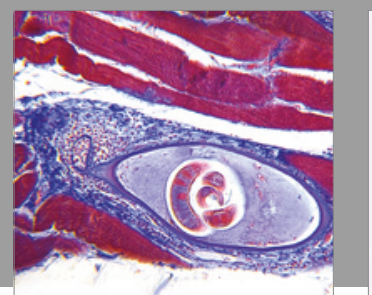

Gastroenterology Research and Practice
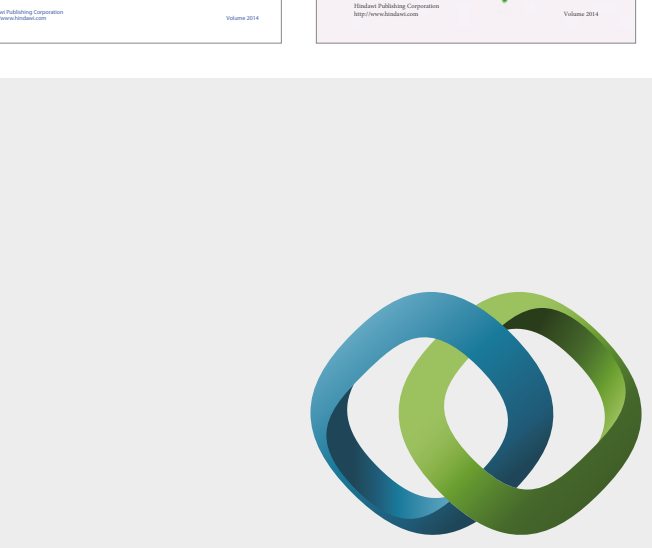

\section{Hindawi}

Submit your manuscripts at

https://www.hindawi.com
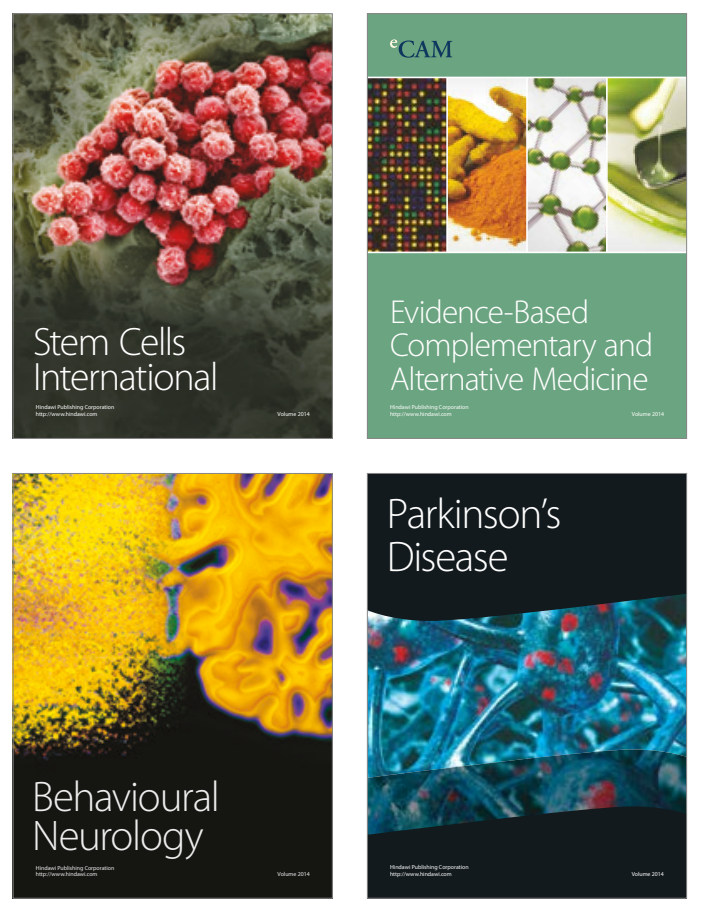
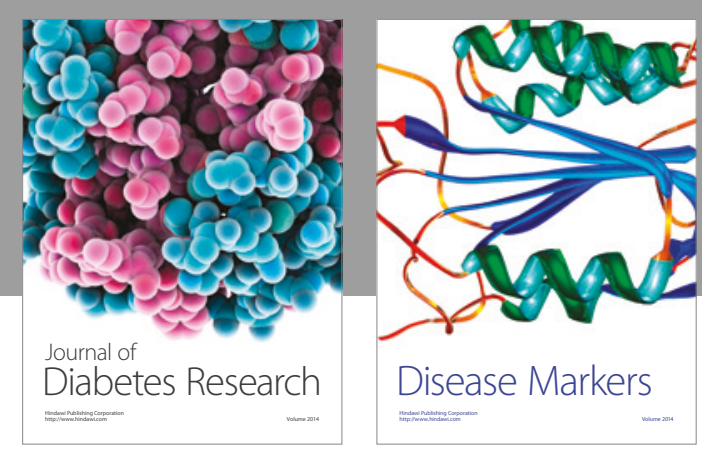

Disease Markers
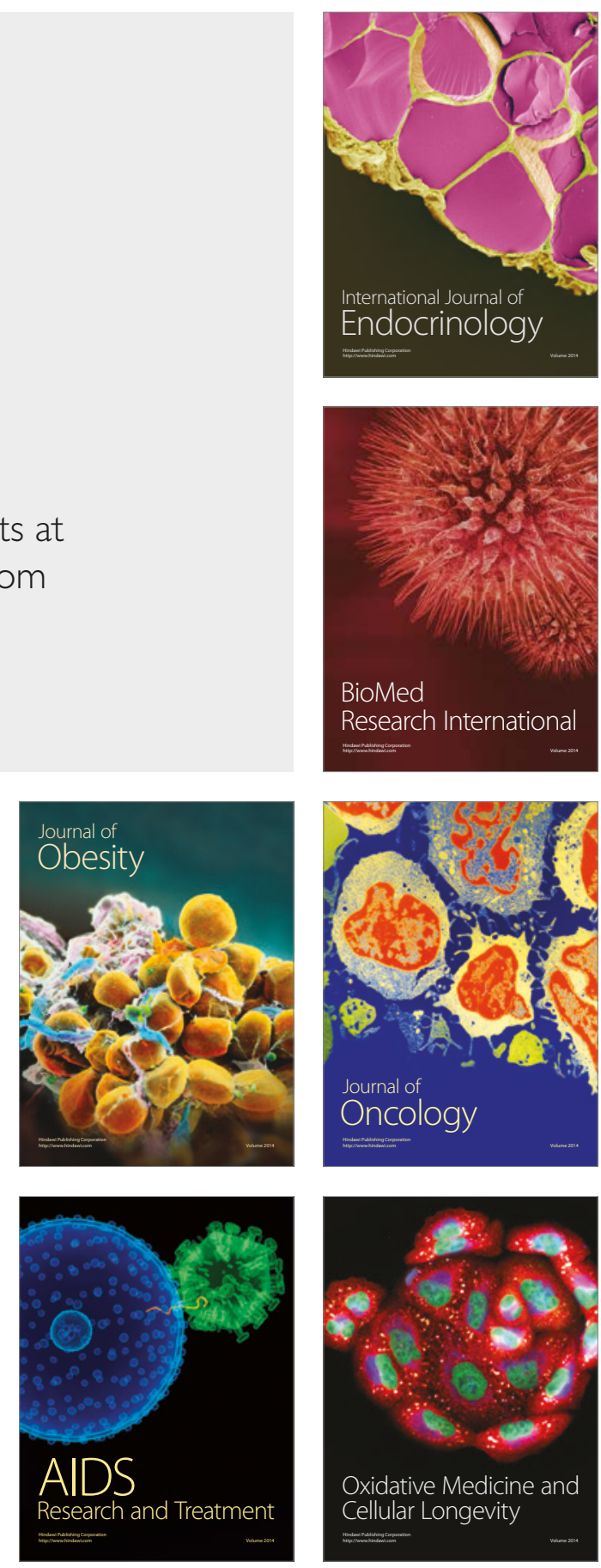